\title{
Dynamic Image Analysis of Glass Fibers as Industrial Fillers and Understanding the Influence of Processing Conditions on the Fiber Length and the Mechanical Properties of Polymers
}

\author{
P. Bajaj ${ }^{1}$ and C. Strom ${ }^{1}$ \\ 1. Sabic, Selkirk, New York 12158 USA
}

Glass fibers among other fillers are routinely incorporated as reinforcements in thermo engineering plastics. The particle size of the incorporated filler affects the polymer's physical properties, such as shrinkage, modulus, heat distortion temperature, impact etc. [1]. The focus of this study is to develop a robust method for determining the length distribution of glass fibers. The effect of processing conditions, specifically screw mixing speed on the fiber length retention and the effect on \% shrinkage in molded parts along the flow direction is studied. Shrinkage is defined as the reduction in size of a molded material in any direction. It is directly related to the flow and cooling conditions under which the material is processed in injection molding. It is an important factor in determining the quality of injection molded parts [2]. The specification range for \% shrinkage is $0.3-0.7$.

$$
\begin{array}{ll}
\text { Shrinkage }(\%)=[(\mathrm{A}-\mathrm{B}) / \mathrm{A}] \times 100 \\
\text { where } & \mathrm{A}=\text { cavity dimension, } \mathrm{mm} \text { (in) } \\
& \mathrm{B}=\text { dimension of molded specimen, } \mathrm{mm} \text { (in) }
\end{array}
$$

Conventional methods for glass fiber length analysis are based on a static imaging that combines optical or scanning electron microscopy (SEM) imaging followed by image processing and analysis to return quantitative data (Figure a). These methods are time consuming, tedious, highly dependent on sample preparation and sample size (Figure a, b). In contrast, the dynamic imaging technique provides simultaneous measurement of particle size. It uses large sample volumes to generate robust statistics based on random orientation of particles.

Method development includes details on the effect of sample pre-conditioning, use of size and shape filters and validation. Intersecting fibers can be automatically rejected by setting a size filter based on the known width of the fibers (anything larger than the known width must be the result of either intersecting or adjacent fibers) and small agglomerates from ash residues can be rejected by including a shape filter based on aspect ratio. The simplicity of the dynamic imaging method can be seen by comparing Figure $b$ and Figure c. Using mild air dispersion pressure $(20 \mathrm{kPa})$ fibers are continuously monitored using a high resolution camera system and real time statistical distribution of size is generated. Figure d, shows the overlay of the measured length distribution from three runs on a test sample. Table 1 summarizes the data from the ANOVA analysis showing the repeatability of glass fiber measurements using dynamic image analysis $(p$ value $=0.765)$ (Figure e). Note that approximately 10,000 fibers were measured for each sample and that it took only 2 to 3 minutes for each sample with this dynamic method.

Next, the effect of processing conditions, specifically screw mixing speed on the fiber length retention was studied. At higher screw speeds, more breakage of fibers is expected due to increased injection force (Figure f, g). Box plot from the ANOVA analysis shows the statistical differences in glass fiber length $(\mu \mathrm{m})$ as a function of screw mixing speed $(\mathrm{rpm})(p$ value $=0.00)$ (Figure $\mathrm{h})$. This effect translates into mechanical properties as seen in loss of shrinkage measured in flow direction of molded parts for shorter fibers at screw speed up to $340 \mathrm{rpm}$ (Figure i, Table 2). The glass fiber reinforcement in 
polymers generally shows anisotropic properties. At screw mixing speed of $340 \mathrm{rpm}$, glass fibers undergo more attrition resulting in \% shrinkage to 0.81 compared to other mixing conditions. This could be explained due to random alignment of the shorter fibers bringing isotropy to the system and making the part small.

In conclusions, it is evident that the understanding of structure-function correlation between fiber length and the \% shrinkage of molded part as a function of screw mixing speed is critical in defining the optimized processing conditions for the glass fiber filled polymeric material and improve product performance.

\section{References:}

[1] A. Jillavenkatesa et al., NIST Practice Guide, 960 (2001), p.1.

[2] Chang, T.C., Polym. Eng. Sci., 41, 2001, 703.
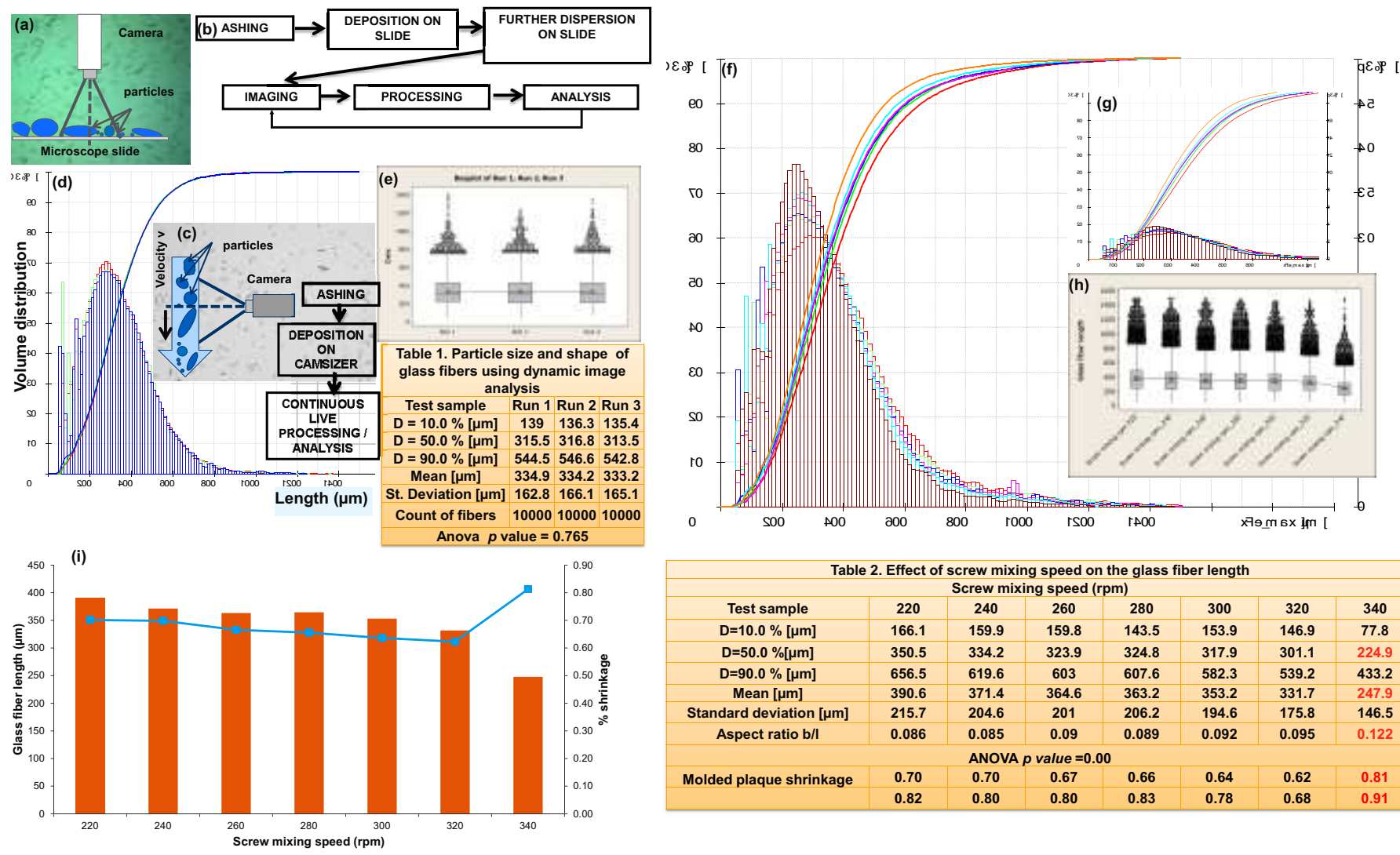

\begin{tabular}{|c|c|c|c|c|c|c|c|}
\hline \multicolumn{8}{|c|}{ Screw mixing speed $(\mathrm{rpm})$} \\
\hline Test sample & 220 & 240 & 260 & 280 & 300 & 320 & 340 \\
\hline$D=10.0 \%[\mu \mathrm{m}]$ & 166.1 & 159.9 & 159.8 & 143.5 & 153.9 & 146.9 & 77.8 \\
\hline $\mathrm{D}=50.0 \%[\mu \mathrm{m}]$ & 350.5 & 334.2 & 323.9 & 324.8 & 317.9 & 301.1 & 224.9 \\
\hline $\mathrm{D}=90.0 \%[\mu \mathrm{m}]$ & 656.5 & 619.6 & 603 & 607.6 & 582.3 & 539.2 & 433.2 \\
\hline Mean $[\mu \mathrm{m}]$ & 390.6 & 371.4 & 364.6 & 363.2 & 353.2 & 331.7 & 247.9 \\
\hline Standard deviation $[\mu \mathrm{m}]$ & 215.7 & 204.6 & 201 & 206.2 & 194.6 & 175.8 & 146.5 \\
\hline Aspect ratio b/l & 0.086 & 0.085 & 0.09 & 0.089 & 0.092 & 0.095 & 0.122 \\
\hline \multicolumn{8}{|c|}{ ANOVA $p$ value $=0.00$} \\
\hline \multirow[t]{2}{*}{ Molded plaque shrinkage } & 0.70 & 0.70 & 0.67 & 0.66 & 0.64 & 0.62 & 0.81 \\
\hline & 0.82 & 0.80 & 0.80 & 0.83 & 0.78 & 0.68 & 0.91 \\
\hline
\end{tabular}

Figure (a) Static imaging mode based on optical microscopy. (b) Steps of Static Imaging. (c) Steps of Dynamic imaging mode. (d) Particle size (fiber length) distribution on a test sample for method development. (e) Box plot from the ANOVA analysis of three runs on a test sample showing repeatability of the measurement system (f) Plot showing the effect of screw mixing speed on glass fiber length retention $(\mathrm{g})$ zoom in on the distribution showing the decrease in fiber length with increasing screw mixing speed (rpm). (h) Box plot from the ANOVA analysis showing the significant statistical differences in glass fiber length $(\mu \mathrm{m})$ as a function of screw mixing speed (rpm) ( $p$ value $=0.00)$. (i) Structure-property correlation between glass fiber lengths vs. shrinkage measurements of the molded parts. 